\title{
The Effect of Improving Preoperative Sleep Quality on Perioperative Pain by Zolpidem in Patients Undergoing Laparoscopic Colorectal Surgery: A Prospective, Randomized Study
}

\author{
Zhennan Xiao $\mathbb{D}^{1},{ }^{1}$ Bo Long $\mathbb{D}^{1}$, and Zeji Zhao $\mathbb{D}^{2}$ \\ ${ }^{1}$ Department of Anesthesiology, Shengjing Hospital of China Medical University, Shenyang, Liaoning, China \\ ${ }^{2}$ Department of Anesthesiology, Shenzhen Traditional Chinese Medicine Hospital, Shenzhen, Guangdong, China \\ Correspondence should be addressed to Bo Long; longb@sj-hospital.org
}

Received 18 July 2021; Revised 15 November 2021; Accepted 22 December 2021; Published 11 January 2022

Academic Editor: Parisa Gazerani

Copyright ( 2022 Zhennan Xiao et al. This is an open access article distributed under the Creative Commons Attribution License, which permits unrestricted use, distribution, and reproduction in any medium, provided the original work is properly cited.

\begin{abstract}
Background and Objectives. Opioids are essential in pain management after laparoscopic colorectal surgery while large dose may induce constipation and pneumonia. Ample evidence has demonstrated that postoperative analgesia can improve sleep quality. But the effects of improvement in sleep quality on postoperative pain have yet to be determined. The aim of this study was to investigate the effect of improving preoperative sleep quality by zolpidem on intraoperative analgesia and postoperative pain. Methods. A prospective, randomized study was conducted with 88 patients undergoing laparoscopic colorectal surgery. The experimental group ( $\mathrm{S}$ group, $n=44$ ) was given $10 \mathrm{mg}$ of zolpidem tartrate one night before the surgical procedure, while no medication was given to the control group (C group, $n=44$ ). The primary outcome was the intraoperative remifentanil consumption. Sufentanil consumption, average patient-controlled analgesia (PCA) effective press times, the visual analog scale (VAS) scores, and incidences of postoperative nausea and vomiting (PONV) were recorded at $6 \mathrm{~h}$ (T1), 12 h (T2), and 24h (T3) postoperatively. Results. The intraoperative remifentanil consumption was significantly lower in the $\mathrm{S}$ group than that in the $\mathrm{C}$ group $(p<0.01)$. Sufentanil consumption at $6 \mathrm{~h}$ and $12 \mathrm{~h}$ postoperatively was significantly lower in the $\mathrm{S}$ group than that in the $\mathrm{C}$ group ( $p<0.05$ ); average PCA effective press times and VAS scores, at $6 \mathrm{~h}$ and $12 \mathrm{~h}$ postoperatively, were significantly lower in the $\mathrm{S}$ group than those in the $\mathrm{C}$ group $(p<0.01)$; differences between groups $24 \mathrm{~h}$ postoperatively were not significant. No significant between-group difference was noted in the incidence of nausea and vomiting. Conclusion. Improving patients' sleep quality the night before surgical procedure by zolpidem can decrease the usage of intraoperative analgesics and reduce postoperative pain.
\end{abstract}

\section{Introduction}

Colorectal cancer, with a 5 -year survival rate of $32 \%$, is the fifth leading cause of death in the Chinese population [1]. It is among the top 3 cancers that cause the world's total disability-adjusted life year (DALY) disease burden (the other 2 are lung cancer and breast cancer) [2]. In China, the incidence of colorectal cancer increases significantly with age, and older individuals have more difficulty adjusting their sleep to environmental changes [3,4]. Besides, the presence of sleep disturbance is highly prevalent among the elderly [5]. It is well known that sleep patterns change with age [6] and vary in patients with cancer [7]. Further, cancer and cancer treatments are factors contributing to sleep disturbance [8]. Sleep disturbance is a symptom characterized by impaired sleep quality with abnormal sleep duration [9]. It can lead to poor sleep quality [10], which is considered a risk factor for many conditions, including cardiovascular disease, dementia, obesity, diabetes, depression, pain, and mortality [11-15].

Recent progress has been made in the study of the relationship between preoperative sleep quality and postoperative pain [16-19]. For instance, sleep quality is linked to the development of chronic pain [20,21]. Sleep deprivation 
and sleep disruption can lead to pain and hyperalgesia [22-26]. In addition, many studies have shown that pain and sleep have bidirectional interactions; that is, pain may lead to sleep disturbances that result in poor sleep quality, and, in turn, sleep disturbances can cause increased pain and hyperalgesia $[22,27,28]$. Further, some studies found that sleep disturbance may be a stronger predictor of pain than pain is a predictor of sleep [24, 29-32]. Despite ample evidence that postoperative analgesia can improve sleep quality [33-36], there is limited evidence that an improvement in sleep quality contributes to pain relief.

The aim of this prospective, randomized, single-center study was to evaluate the effect of improving preoperative sleep quality on perioperative pain by administering zolpidem. We hypothesized that administration of zolpidem the night before the procedure would improve preoperative sleep quality, reduce intraoperative anesthetic medication, and contribute to postoperative pain relief.

\section{Materials and Methods}

2.1. Study Design. The study was approved by the Human Research Ethical Committee of Shengjing Hospital, Shenyang, Liaoning Province, China (IRB registration number: 2018PS385K) and registered at https://www.chictr.org.cn (ChiCTR1900022235, March 31, 2019). It was conducted in accordance with the Declaration of Helsinki. Written informed consent was obtained from all participants. This randomized, single-blind study was conducted at the Department of Anesthesiology at Shengjing Hospital of China Medical University, a tertiary referral hospital.

We included consecutive adult patients (age range, 40-70 years) classified as American Society of Anesthesiologists (ASA) physical status I or II who underwent laparoscopic colorectal surgery between March 2019 and October 2019. Exclusion criteria were patient age $<40$ years or $>70$ years; Pittsburgh Sleep Quality Index (PSQI) score $\leq 7$ points; and history of sleep apnea, drug allergy, drug abuse, hepatorenal dysfunction, psychosis, or neuropathy. Patients with a severe hearing impairment or an inability to comprehend or complete the PSQI questionnaire were likewise excluded because of possible barriers to communicating with the anesthesiologists.

2.2. Study Intervention. An anesthesiologist, who did not participate in the surgical procedure, used the Pittsburgh Sleep Quality Index (PSQI) to assess each patient's sleep quality. The PSQI is a self-reported questionnaire used in the measurement of sleep quality over a month [37]. The questionnaire contains 19 items that consist of seven components (subjective sleep quality, sleep latency, sleep duration, sleep efficiency, sleep disturbances, use of sleeping medication, and daytime dysfunction). The score ranges from 0 (no difficulty) to 3 (severe difficulty) for each component. Therefore, overall PSQI scores range from 0 to 21, with a higher score indicating a worse sleep quality. A global PSQI score $>7$ was the cut-off number to indicate poor sleep quality in a Chinese population [38].
All patients with a global PSQI score $>7$ were enrolled in this study and randomly assigned to either the $S$ (zolpidem tartrate) or C (blank control) group by an investigator, with no clinical involvement, using a head-tail binary result coin toss. The head side of the coin indicated the S group (zolpidem tartrate), and the text or tail side of the coin indicated the $\mathrm{C}$ group (blank control). Allocation concealment was ensured by concealing assignments into sealed opaque envelopes that were numbered sequentially and opened the night before the procedure. Zolpidem tartrate (Sanofi Pharmaceutical Co., Ltd., Hangzhou, China) $10 \mathrm{mg}$ was given to patients in the $S$ group the night before the surgical procedure by a nurse independent of the study, while $\mathrm{C}$ group patients were not given medication. The patients in each group were blinded to the existence of the other group. Research personnel were maintained blinded throughout the study.

On arrival in the operating room, all patients were given the Leeds Sleep Evaluation Questionnaire (LSEQ) to evaluate their sleep quality the previous night. The LSEQ is a selfrating scale comprising 10 questions, scored on a $100 \mathrm{~mm}$ visual analog scale, that pertain to 4 domains: the ease of getting to sleep (GTS, items 1-3), the quality of sleep (QOS, items 4 and 5), waking from sleep (AFS, items 6 and 7), and behavior following wakefulness (BFW, items 8-10) [39]. LSEQ is a suitable tool for comparative assessment of the effect of the drug intervention on sleep quality over a short time. It is a reliable and sensitive measurement of the effects of hypnotics on sleep and early morning behavior [40]. Cronbach's alpha coefficients ranged between 0.78 and 0.92 [41]. The LSEQ has proven reliable and sensitive in numerous studies involving people with insomnia aged 55 and older [42]. Patients were self-rated by placing a mark on the line, with the position of the mark indicating the extent of the change. Higher scores indicate better sleep on a scale of 0 to 100 (corresponding from impairment to improvement). An anesthesiologist who did not participate in the surgical procedure was responsible for recording the LSEQ scores.

2.3. Anesthesia Protocol. Intravenous (IV) access and standard monitoring with an electrocardiogram (ECG; lead II), heart rate (HR), blood pressure (BP), and pulse oximetry were established. Peripheral arterial catheters were inserted for intraoperative monitoring of mean arterial pressure (MAP), and a multifunction combination monitor (HXD-1 Beijing Easymonitor Technology Co., Ltd.) was routinely connected to monitor the antinociceptive state and depth of anesthesia.

Once all standard monitors were connected to the patient, general anesthesia was induced by administration of IV sufentanil $(0.5 \mu \mathrm{g} / \mathrm{kg})$ and propofol $(1.5 \mathrm{mg} / \mathrm{kg})$. Orotracheal intubation was achieved using a cuffed tube and facilitated by cisatracurium $(0.2 \mathrm{mg} / \mathrm{kg})$. Following induction and intubation, ventilation (Drager; Primus, Germany) was performed at tidal volumes of 8 to $10 \mathrm{~mL} / \mathrm{kg}$, based on ideal body weight, with a $1: 2$ inspiratory-to-expiratory time ratio. Fresh gas was set to a $50 \%$ oxygen-nitrous oxide mixed flow at $2 \mathrm{~L} / \mathrm{min}$. Ventilator frequency was initially set at 12 
breaths/min and was adjusted as needed to maintain an endtidal carbon dioxide tension $\left(\mathrm{ETCO}_{2}\right)$, assessed by blood gas analysis, of 35 to $45 \mathrm{~mm} \mathrm{Hg}$ throughout the procedure.

Remifentanil $(1.5-6 \mu \mathrm{g} / \mathrm{kg} / \mathrm{h})$ and propofol (4-10 mg/kg/ h), administered continuously by a syringe pump (Zhejiang University Medical Instrument Co., Ltd., Beijing, China), were used for maintenance of general anesthesia. The pain threshold index (PTI) and wavelet index (WLI) were monitored with a multifunction combination monitor (HXD-1 Beijing Easymonitor Technology Co., Ltd.). According to the manufacturer's recommendations, PTI and WLI values of 40 to 60 were maintained to ensure adequate depths of anesthesia during general anesthesia. A single IV bolus of sufentanil $(0.1 \mu \mathrm{g} / \mathrm{kg})$ and ketorolac tromethamine (30 mg IV) was given to all patients $30 \mathrm{~min}$ before the end of surgery as prophylaxis against postoperative pain, along with ramosetron hydrochloride $(0.3 \mathrm{mg}$ IV) to curb postoperative nausea and vomiting.

After extubation, patients were transferred to the postanesthesia care unit and provided with a patient-controlled analgesia pump (Royal Fornia Medical Equipment Co., Ltd., Shenzhen, China). The pump was prepared at a total volume of $100 \mathrm{ml}$ by mixing $2 \mu \mathrm{g} / \mathrm{kg}$ sufentanil in normal saline. The background infusion rate and a demand dose were $2 \mathrm{ml} / \mathrm{h}$ and $1 \mathrm{ml}$, respectively.

Another anesthesiologist who was blind to the research protocol was responsible for recording the amount of remifentanil consumed during the surgical procedure. A nurse who was blind to the operative details used a visual analog scale (VAS), with 0 corresponding to no pain and 10 to maximum pain, to assess each patient's pain intensity during rest and movement stages at the following postoperative time $(T)$ intervals: T1 $(6 \mathrm{~h}), \mathrm{T} 2(12 \mathrm{~h})$, and T3 $(24 \mathrm{~h})$. The amount of sufentanil consumed within $24 \mathrm{~h}$ of the procedure was also recorded at T1, T2, and T3. Average PCA effective press times and incidence of postoperative nausea and vomiting were also recorded within $24 \mathrm{~h}$ of the surgical procedure.

2.4. Sample Size. According to our previous 20-patient pilot study, the mean intraoperative remifentanil consumption was $0.90 \pm 0.35 \mathrm{mg}$ in the patients who received $10 \mathrm{mg}$ of zolpidem tartrate one night before the surgical procedure and $1.20 \pm 0.51 \mathrm{mg}$ in the control group. The study was powered to detect a reduction of $0.30 \mathrm{mg}$ in the intraoperative remifentanil consumption. Based on a power of $80 \%$ and alpha $=0.05$, we calculated that a sample size of 70 (35 in each group) was required. Finally, a sample size of 88 (44 in each group) was required to account for $20 \%$ dropout rate.

2.5. Statistical Analysis. Descriptive statistics are reported as means with standard deviation (SD) or medians (interquartile range) for continuous variables and as frequencies or proportions for categorical variables. Age, weight, operation time, PSQI score, intraoperative remifentanil consumption, postoperative sufentanil consumption, PCA press times, and VAS scores were compared by the independent $t$-test or Mann-Whitney $U$ test; and gender, ASA status, and incidence of postoperative nausea and vomiting were analyzed using either the chi-squared or Fisher's exact test, as appropriate. All statistical analyses were performed with standard software (SPSS v22.0; SPSS Inc., Chicago, IL, USA) and statistical significance was set at $p<0.05$.

\section{Results}

From March 2019 to October 2019, a total of 88 patients were enrolled in the study. The CONSORT diagram for patient recruitment is shown in Figure 1. Two cases were eliminated due to poor electrode-skin contact or noise interference, and another three cases were excluded due to transfer to the ICU. Patient characteristics and intraoperative data were similar in the two groups (Table 1). A significant difference was found in the LSEQ scores on the morning of the surgical procedure. The sleep quality in the $S$ group was improved after administration of zolpidem. Detailed information is shown in Table 2.

Regarding the primary outcome, the intraoperative remifentanil consumption was significantly lower in the $\mathrm{S}$ group than in the $\mathrm{C}$ group $(0.84[0.61-1.11]$ vs. 1.00 [0.88-1.55], $p<0.01$ ) (Table 3). In the first 24 hours postoperatively, the sufentanil consumption at $6 \mathrm{~h}$ and $12 \mathrm{~h}$ was significantly lower in the $S$ group than that in the $C$ group ( 6 h: $17.51 \pm 3.09$ vs. $20.32 \pm 5.07, p<0.05 ; 12$ h: $34.35 \pm 5.57$ vs. $38.78 \pm 8.04, p<0.01)$, but no between-group difference occurred at $24 \mathrm{~h}(p=0.064)$ (Table 4$)$. Similarly, average PCA effective press time was significantly lower in the $S$ group than that in the $\mathrm{C}$ group at $6 \mathrm{~h}(1[1-2]$ vs. $3[1-4]$, $p=0.001)$ and $12 \mathrm{~h}(2[1-3.25]$ vs. 4 [2.5-5], $p<0.001)$ postoperatively, but no between-group difference occurred at $24 \mathrm{~h}(p=0.159)$ (Table 5). In, addition, patients in the $S$ group had lower VAS scores $(p<0.01)$ at $6 \mathrm{~h}$ and $12 \mathrm{~h}$ postoperatively compared to the $\mathrm{C}$ group, both at rest and during movement ( $6 \mathrm{~h}$ rest: $2.54 \pm 0.95$ vs. $1.62 \pm 0.99$, $p<0.001$; 6 h movement: $3.51 \pm 1.36$ vs. $2.83 \pm 1.15, p<0.01$; 12 h rest: $3.78 \pm 1.04$ vs. $3.00 \pm 1.21, p<0.01 ; 12$ h movement: $4.80 \pm 1.15$ vs. $3.98 \pm 1.24, p<0.01)$. However, the betweengroup differences at $24 \mathrm{~h}$ postoperatively were not significant ( 24 h rest: $p=0.066 ; 24$ h movement: $p=0.069$ ) (Table 6). No significant between-group differences were noted in the incidence of nausea and vomiting between the 2 groups ( $6 \mathrm{~h}$ : $p=0.625,12 \mathrm{~h}: p=1,24 \mathrm{~h}: p=0.738$ ) (Table 7).

\section{Discussion}

The results of this study showed that administering a dose of zolpidem $(10 \mathrm{mg}$ ) can improve sleep quality preoperatively and reduce the amount of analgesics used intraoperatively and postoperatively. Moreover, lower pain intensity, both at rest and during movement, was noticed in patients given zolpidem compared to patients in the control group within the first $12 \mathrm{~h}$ after surgery. However, no significant difference was found in the incidence of PONV and length of hospital stay between treatment and control group patients. 


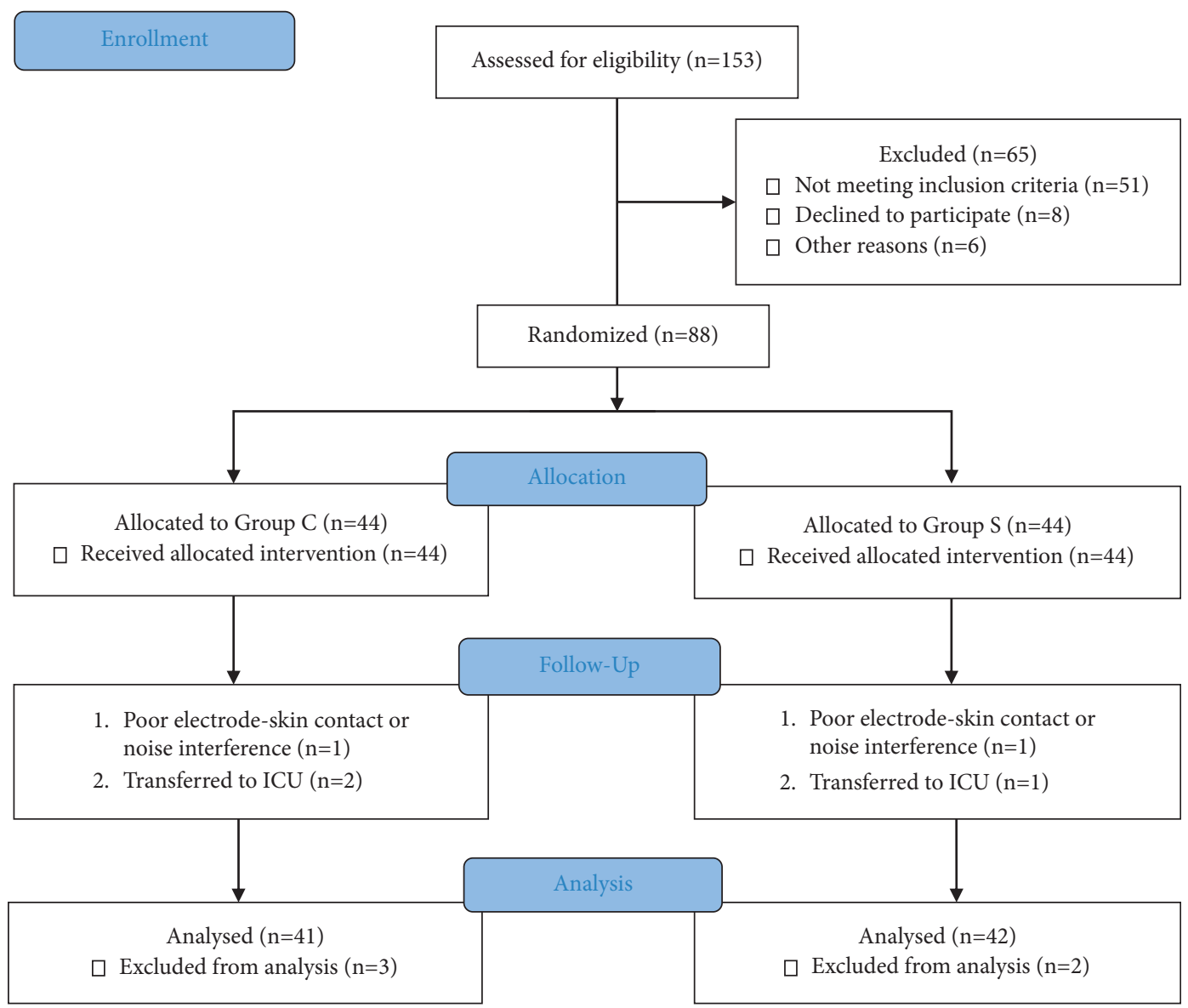

FIGURE 1: Consolidated standards of reporting trials (CONSORT) flow diagram.

TABle 1: Patient characteristics and intraoperative data.

\begin{tabular}{lcc}
\hline & Group C & Group S \\
\hline Gender (M/F) & $21 / 20$ & $23 / 19$ \\
ASA status (I/II) & $17 / 24$ & $16 / 26$ \\
Age (years) & $60.34 \pm 7.02$ & $60.02 \pm 7.32$ \\
Weight (kg) & $66.83 \pm 11.07$ & $65.48 \pm 10.71$ \\
PSQI & $9.22 \pm 1.81$ & $8.98 \pm 1.62$ \\
Operation time (min) & $205.29 \pm 35.46$ & $192.43 \pm 33.36$ \\
Hospitalization time (days) & $19.90 \pm 3.07$ & $20.81 \pm 2.04$ \\
\hline
\end{tabular}

Note: data are expressed as mean \pm SD. Abbreviations: PSQI: Pittsburgh Sleep Quality Index; group C: blank control; group S: zolpidem tartrate.

TABLE 2: Leeds Sleep Evaluation Questionnaire (LSEQ) scores.

\begin{tabular}{lccc}
\hline & Group C & Group S & $p$ value \\
\hline GTS & $45.20 \pm 8.71$ & $76.55 \pm 7.63^{*}$ & $<0.001$ \\
QOS & $46.44 \pm 9.35$ & $74.29 \pm 7.46^{*}$ & $<0.001$ \\
AFS & $45.90 \pm 13.08$ & $51.48 \pm 15.90$ & 0.085 \\
BFW & $52.10 \pm 12.49$ & $61.76 \pm 10.99^{*}$ & $<0.001$ \\
\hline
\end{tabular}

Note: data are expressed as mean \pm SD. ${ }^{*} p<0.05$ vs. group C. Abbreviations: GTS: getting to sleep; QOS: quality of sleep; AFS: awakening from sleep; BFW: behavior following wakefulness. Group C: blank control; group S: zolpidem tartrate.

Sleep is a physiological phenomenon which is indispensable to an individual's physical and mental health $[43,44]$. However, almost half of older adults in China
TABLE 3: Intraoperative remifentanil consumption.

\begin{tabular}{lccc}
\hline & Group C & Group S & $\begin{array}{c}p \\
\text { value }\end{array}$ \\
\hline $\begin{array}{l}\text { Remifentanil dose } \\
(\mathrm{mg})\end{array}$ & $\begin{array}{c}1.00 \\
(0.88-1.55)\end{array}$ & $\begin{array}{c}0.84 \\
(0.61-1.11)^{*}\end{array}$ & 0.002 \\
\hline
\end{tabular}

Note: data expressed as median (interquartile range). ${ }^{*} p<0.05$ vs. group

C. Abbreviations: group C: blank control; group S: zolpidem tartrate.

experience various degrees of sleep disturbance [45, 46]. Previous investigators have speculated that poor sleep quality predicts high next-day pain intensity that may persist up to 2 weeks after surgery [47]. Another published review concluded that behavioral sleep interventions can produce improvements in sleep that are beneficial to pain outcomes 
Table 4: Postoperative sufentanil consumption.

\begin{tabular}{lccc}
\hline & Group C & Group S & $p$ value \\
\hline Postoperative 6 h (ug) & $20.32 \pm 5.07$ & $17.51 \pm 3.09^{*}$ & 0.016 \\
Postoperative 12 h (ug) & $38.78 \pm 8.04$ & $34.35 \pm 5.57^{*}$ & 0.004 \\
Postoperative 24 h (ug) & $73.68 \pm 14.69$ & $68.41 \pm 10.58$ & 0.064 \\
\hline
\end{tabular}

Note: data expressed as means \pm SD. ${ }^{*} p<0.05$ vs. group C. Abbreviations: group C: blank control; group S: zolpidem tartrate.

TABle 5: Average PCA effective press times.

\begin{tabular}{lccc}
\hline & Group C & Group S & $p$ value \\
\hline Postoperative 6h & $3(1-4)$ & $1(1-2)^{*}$ & 0.001 \\
Postoperative 12 $\mathrm{h}$ & $4(2.5-5)$ & $2(1-3.25)^{*}$ & $<0.001$ \\
Postoperative 24 h & $5.68 \pm 4.25$ & $4.52 \pm 3.10$ & 0.159 \\
\hline
\end{tabular}

Note: data expressed as means $\pm \mathrm{SD}$ and median (interquartile range). ${ }^{*} p<0.05$ vs. group C. Abbreviations: group C: blank control; group S: zolpidem tartrate.

TABle 6: Postoperative VAS.

\begin{tabular}{lccc}
\hline & Group C & Group S & $p$ value \\
\hline PO6 (rest) & $2.54 \pm 0.95$ & $1.62 \pm 0.99^{*}$ & $<0.001$ \\
PO6 (movement) & $3.51 \pm 1.36$ & $2.83 \pm 1.15^{*}$ & 0.016 \\
PO12 (rest) & $3.78 \pm 1.04$ & $3.00 \pm 1.21^{*}$ & 0.002 \\
PO12 (movement) & $4.80 \pm 1.15$ & $3.98 \pm 1.24^{*}$ & 0.002 \\
PO24 (rest) & $4(3.5-5)$ & $4(3-4)$ & 0.066 \\
PO24 (movement) & $5(4-6)$ & $4(4-5)$ & 0.069 \\
\hline
\end{tabular}

Note: data expressed as means $\pm \mathrm{SD}$ and median (interquartile range). ${ }^{*} p<0.05$ vs. group C. Abbreviations: PO6: postoperative $6 \mathrm{~h}$; PO12: postoperative $12 \mathrm{~h}$; PO24: postoperative $24 \mathrm{~h}$. Group C: blank control; group S: zolpidem tartrate.

TABLE 7: Nausea and vomiting.

\begin{tabular}{lcc}
\hline & Group C & Group S \\
\hline Postoperative $6 \mathrm{~h}$ & 12 & 10 \\
Postoperative $12 \mathrm{~h}$ & 8 & 8 \\
Postoperative $24 \mathrm{~h}$ & 5 & 4 \\
\hline
\end{tabular}

Note: values are $n$ (\%). Abbreviations: group C: blank control; group S: zolpidem tartrate.

in middle-aged or older adults [48]. Consequently, it may be worthwhile to relieve postoperative pain by improving sleep quality.

In the current study, the patients in the $S$ group achieved higher LSEQ scores the following morning after receiving a dose of zolpidem $(10 \mathrm{mg})$. This finding suggests that zolpidem can significantly increase sleep quality in patients with sleep disturbance. Zolpidem, an alternative to benzodiazepines, is a prescription sedative-hypnotic drug with a well-established safety profile [49]. It improves sleep quality by helping patients fall asleep quickly and producing a more natural sleep pattern [50]. Although some studies have reported that hypnotics improve GTS and QOS but produce a dose-dependent "hangover" effect that results in impairment in AFS [37], we did not find any difference in AFS in the S group compared with the $\mathrm{C}$ group. A possible explanation is that we only administered a single $10 \mathrm{mg}$ dose of zolpidem one day before the surgical procedure, which is a much lower dosage than in previous studies. Furthermore, zolpidem is one of the nonbenzodiazepine hypnotics with a short halflife and low incidence of residual effects on alertness the following morning.

Regarding the primary outcome, our study revealed that patients with better sleep quality were more likely to have lower analgesics consumption during the surgical procedure. In clinical practice, the administration of antinociceptive analgesics is usually determined by the anesthesiologist's clinical experience [51]. Therefore, it is challenging to reach an appropriate analgesic dosage without effective or objective evaluation tools. To support the proper use of analgesics, we applied PTI to reflect the antinociceptive state in order to guide the administration of opioids under general anesthesia. PTI is a newly developed EEG-derived parameter that can be used to predict the response to, rather than detecting the result of, noxious stimulation. Wu et al. demonstrated that the PTI is proven to be effective and subtle in predicting the nociceptive response induced by noxious stimuli during general anesthesia [52]. The principle of the PTI calculation was reported in China Medical Engineering in 2017 [53]. In this study, lower remifentanil consumption was reported in the $\mathrm{S}$ compared to $\mathrm{C}$ group. To our knowledge, only limited research has been conducted to show that improved sleep quality can reduce the amount of analgesics administered intraoperatively.

Furthermore, patients with better sleep quality had significantly lower sufentanil consumption, less average PCA effective press times, and lower VAS scores at $6 \mathrm{~h}$ and $12 \mathrm{~h}$ postoperatively, compared to the $\mathrm{C}$ group. Our result is partially consistent with that of a systematic review showing that perioperative administration of sleep-promoting drugs can improve pain relief [54]. Similarly, Shakya et al. found that zolpidem $10 \mathrm{mg}$, administered from 2 days preoperatively to 5 days after surgery, can improve sleep quality, relieve pain, and promote recovery in patients underwent total hip arthroplasty [55]. Another similar result was reported by Gong et al. in a study involving 148 patients who underwent total knee arthroplasty. They found a greater improvement in sleep quality after taking zolpidem for 2 weeks, with decreased postoperative VAS pain scores and analgesics consumption and a better quality of life [56].

However, there were several differences between their studies and ours. Firstly, in contrast with their studies in orthopedic patients, we mainly focused on the patients with colorectal cancer. Reportedly, nearly $40 \%$ of patients with colorectal cancer in China have preoperative insomnia [57]. A case-control study demonstrated that better sleep quality is protective for patients with colorectal cancer [58]. Secondly, patients undergoing laparoscopic colorectal surgery are more subjected to visceral pain rather than somatic pain. Visceral pain is defined as a deep, dull, and vague sensation that often cannot be described or is poorly localized [59]. Acute visceral pain may develop into chronic visceral pain that results in behavioral symptoms such as anxiety, fear, and depression [60]. In addition, opioids are commonly used in the management of acute visceral pain; however, 
large doses of opioids may induce constipation that is associated with a series of physical and psychological symptoms as well as a poor quality of life [61]. Therefore, it is crucial for patients with colorectal cancer to have better pain relief and a reduction in opioid requirements.

Notably, our finding suggested that improved sleep quality can reduce the amount of analgesics used both intraoperatively and within the first $12 \mathrm{~h}$ postoperatively. A possible explanation lies in the mechanisms underlying the relationship between sleep and pain, including endogenous pain modulation, inflammation, affection, and mood as well as some roles of different endogenous substances [62]. Endogenous pain modulation refers to the series of actions that affect nociceptive signal processing central nervous system. Ample clinical evidence shows that sleep fragmentation and deprivation can interfere or even inhibit the descending pain inhibitory system, thus leading to an increase in pain intensity or pain hypersensitivity [63-65]. Moreover, sleep disturbance is associated with central sensitization. Smith et al. demonstrated that sleep disruption enhanced secondary hyperalgesia in males and increased temporal summation in females [66].

Cytokines play critical roles in the development of inflammatory pain. Sleep disturbance can lead to increased levels of proinflammatory cytokines such as IL-1 $\beta$, IL-6, TNF- $\alpha$, and CRP- 6 , which further increase pain intensity and sensitivity and increase the incidence of pain [67-70]. Additionally, individuals who experience poor sleep report higher negative effect (e.g., anxiety, anger, and fear), which is believed to cause hypervigilance and sensitization to pain $[71,72]$. Furthermore, the potential significance of endogenous substances has been elucidated in the mechanism of pain. Roman et al. found that sleep loss can decrease the sensitivity of the serotonin-1A receptor, which is involved in the processing of nociception [73]. Many clinical and experimental studies illustrate that sleep deprivation is related to increased NMDA receptor activation, which is involved in secondary hyperalgesia and temporal summation [74-78]. Sleep deprivation also can downregulate dopamine 2 receptors that are associated with the modulation of acute antinociception [79, 80]. Last, a weak zolpidem-induced analgesic effect through opioidergic mechanisms was observed in a mouse model [81].

Generally speaking, the intraoperative use of remifentanil has been closely linked to postoperative hyperalgesia. Thus, the lower intraoperative remifentanil consumption may contribute to the less postoperative sufentanil consumption and lower pain intensity in the $S$ group, compared to those in the $\mathrm{C}$ group. Nevertheless, a study published in the journal, Anaesthesia [82], confirmed that it is less likely to cause postoperative hyperalgesia when remifentanil infusion rate is below $0.2 \mu \mathrm{g} \cdot \mathrm{kg}^{-1} \cdot \mathrm{min}^{-1}$. In our clinical trial, remifentanil infusion rate was $0.08 \pm 0.02 \mu \mathrm{g} \cdot \mathrm{kg}^{-1} \cdot \mathrm{min}^{-1}$ in the $\mathrm{C}$ group and $0.07 \pm 0.02 \mu \mathrm{g} \cdot \mathrm{kg}^{-1} \cdot \mathrm{min}^{-1}$ in the $\mathrm{S}$ group. Both rates were far less than $0.2 \mu \mathrm{g} \cdot \mathrm{kg}^{-1} \cdot \mathrm{min}^{-1}$. This led us to believe that the difference in postoperative analgesia was mainly due to the impact of improved sleep quality by zolpidem.
Based on the above research, we infer that zolpidem can improve patients' sleep quality preoperatively. This improvement contributes to the reduction in the usage of opioid analgesics intraoperatively and postoperatively (within $12 \mathrm{~h}$ ). Further studies with objective measures (e.g., fMRI and PSG) should be performed to validate our findings.

Our study has some limitations. First, sleep quality was only assessed using the PSQI and LSEQ without polysomnography for evaluating sleep quality, quantity, and architecture. We did not assess patient fatigue and depression, which may also influence the measured outcomes. Second, light and noise may influence sleep quality in hospitalized patients. To minimize the effect of such disruptions, we allocated the patients randomly, thereby distributing the potential confounders equally between the groups. Additionally, as this is only a single-center singleblind trial in a relatively small population, a multicenter trial is necessary to verify its results. We acknowledge this was a single-blind study since patients were not adequately blinded. However, patients in each group were blinded to the existence of the other group. In addition, all researchers taking measurements remained blinded to the details of the study, as were also the data analysts. Blinding was also maintained by randomization and allocation concealment to minimize bias. Finally, we only administered zolpidem the night before the surgical procedure and reported the results within the first $24 \mathrm{~h}$ postoperatively. We did not examine the long-term duration and efficacy of pain control. Future studies should focus on the suitable dosage of zolpidem and any other medical interventions.

\section{Conclusion}

Improving the sleep quality of patients the night before surgery by zolpidem can decrease the usage of intraoperative analgesics and reduce postoperative pain. Given the widely varying available methods, further exploration of suitable and effective ways to improve sleep quality while minimizing side effects is required. The results of this study may provide a new approach to promoting enhanced recovery after surgery.

\section{Data Availability}

The data and materials that support the findings of this study are available from the corresponding author upon reasonable request.

\section{Conflicts of Interest}

The authors declare that there are no conflicts of interest regarding the publication of this paper.

\section{Authors' Contributions}

All authors contributed to data analysis and drafting or revising the article, have agreed on the journal to which the article will be submitted, gave final approval of the version to 
be published, and agree to be accountable for all aspects of the work.

\section{Acknowledgments}

The authors thank patients, their families, and institutions for supporting this study.

\section{References}

[1] L. Yang, D. M. Parkin, L. D. Li, Y. D. Chen, and F. Bray, "Estimation and projection of the national profile of cancer mortality in China: 1991-2005," British Journal of Cancer, vol. 90, no. 11, pp. 2157-2166, 2004.

[2] I. Soerjomataram, J. Lortet-Tieulent, D. M. Parkin et al., "Global burden of cancer in 2008: a systematic analysis of disability-adjusted life-years in 12 world regions," Lancet, vol. 380, no. 9856, pp. 1840-1850, 2012.

[3] S. Rosenberg-Adamsen, H. Kehlet, C. Dodds, and J. Rosenberg, "Postoperative sleep disturbances: mechanisms and clinical implications," British Journal of Anaesthesia, vol. 76, no. 4, pp. 552-559, 1996.

[4] R. Sterniczuk, B. Rusak, and K. Rockwood, "Sleep disturbance in older ICU patients," Clinical Interventions in Aging, vol. 9, pp. 969-977, 2014.

[5] D. Foley, S. Ancoli-Israel, P. Britz, and J. Walsh, "Sleep disturbances and chronic disease in older adults: results of the 2003 National Sleep Foundation Sleep in America Survey," Journal of Psychosomatic Research, vol. 56, no. 5, pp. 497-502, 2004.

[6] A. Schlemmer, U. Parlitz, S. Luther, N. Wessel, and T. Penzel, "Changes of sleep-stage transitions due to ageing and sleep disorder," Philosophical Transactions Series A, Mathematical, Physical, and Engineering Sciences, vol. 373, 2015.

[7] D. Chen, Z. Yin, and B. Fang, "Measurements and status of sleep quality in patients with cancers," Supportive Care in Cancer: Official Journal of the Multinational Association of Supportive Care in Cancer, vol. 26, no. 2, pp. 405-414, 2018.

[8] D. J. Langford, K. Lee, and C. Miaskowski, "Sleep disturbance interventions in oncology patients and family caregivers: a comprehensive review and meta-analysis," Sleep Medicine Reviews, vol. 16, no. 5, pp. 397-414, 2012.

[9] B. Zhu, C. Vincent, M. C. Kapella et al., "Sleep disturbance in people with diabetes: a concept analysis," Journal of Clinical Nursing, vol. 27, no. 1-2, pp. e50-e60, 2018.

[10] B. Izci-Balserak and G. W. Pien, "The relationship and potential mechanistic pathways between sleep disturbances and maternal hyperglycemia," Current Diabetes Reports, vol. 14, no. 2, p. 459, 2014.

[11] J. Yin, X. Jin, Z. Shan et al., "Relationship of sleep duration with all-cause mortality and cardiovascular events: a systematic review and dose-response meta-analysis of prospective cohort studies," Journal of American Heart Association, vol. 6, no. 9, 2017.

[12] L. Shi, S. J. Chen, M. Y. Ma et al., "Sleep disturbances increase the risk of dementia: a systematic review and meta-analysis," Sleep Medicine Reviews, vol. 40, pp. 4-16, 2018.

[13] Y. Fatima, S. A. Doi, and A. A. Mamun, "Sleep quality and obesity in young subjects: a meta-analysis," Obesity Reviews, vol. 17, no. 11, pp. 1154-1166, 2016.

[14] J. E. Gangwisch, S. B. Heymsfield, B. Boden-Albala et al., "Sleep duration as a risk factor for diabetes incidence in a large U.S. sample," Sleep, vol. 30, no. 12, pp. 1667-1673, 2007.
[15] L. Zhai, H. Zhang, and D. Zhang, "Sleep duration and depression among adults: a meta-analysis OF prospective studies," Depression and Anxiety, vol. 32, no. 9, pp. 664-670, 2015.

[16] S. Orbach-Zinger, S. Fireman, A. Ben-Haroush et al., "Preoperative sleep quality predicts postoperative pain after planned caesarean delivery," European Journal of Pain, vol. 21, no. 5, pp. 787-794, 2017.

[17] C. E. Wright, D. H. Bovbjerg, G. H. Montgomery et al., "Disrupted sleep the night before breast surgery is associated with increased postoperative pain," Journal of Pain and Symptom Management, vol. 37, no. 3, pp. 352-362, 2009.

[18] L. Poole, T. Kidd, E. Leigh, A. Ronaldson, M. Jahangiri, and A. Steptoe, "Preoperative sleep complaints are associated with poor physical recovery in the months following cardiac surgery," Annals of Behavioral Medicine, vol. 47, no. 3, pp. 347-357, 2014.

[19] J. A. Rabbitts, C. B. Groenewald, G. G. Tai, and T. M. Palermo, "Presurgical psychosocial predictors of acute postsurgical pain and quality of life in children undergoing major surgery," The Journal of Pain, vol. 16, no. 3, pp. 226-234, 2015.

[20] R. D. Treede, W. Rief, A. Barke et al., "A classification of chronic pain for ICD-11," Pain, vol. 156, no. 6, pp. 1003-1007, 2015.

[21] G. Vanini, "Sleep deprivation and recovery sleep prior to a noxious inflammatory insult influence characteristics and duration of pain," Sleep, vol. 39, no. 1, pp. 133-142, 2016.

[22] S. Lautenbacher, B. Kundermann, and J. C. Krieg, "Sleep deprivation and pain perception," Sleep Medicine Reviews, vol. 10, no. 5, pp. 357-369, 2006.

[23] E. Azevedo, G. M. Manzano, A. Silva, R. Martins, M. L. Andersen, and S. Tufik, "The effects of total and REM sleep deprivation on laser-evoked potential threshold and pain perception," Pain, vol. 152, no. 9, pp. 2052-2058, 2011.

[24] R. R. Edwards, D. M. Almeida, B. Klick, J. A. Haythornthwaite, and M. T. Smith, "Duration of sleep contributes to next-day pain report in the general population," Pain, vol. 137, no. 1, pp. 202-207, 2008.

[25] J. K. Cremeans-Smith, K. Millington, E. Sledjeski, K. Greene, and D. L. Delahanty, "Sleep disruptions mediate the relationship between early postoperative pain and later functioning following total knee replacement surgery," Journal of Behavioral Medicine, vol. 29, no. 2, pp. 215-222, 2006.

[26] F. Chouchou, S. Khoury, J. M. Chauny, R. Denis, and G. J. Lavigne, "Postoperative sleep disruptions: a potential catalyst of acute pain?" Sleep Medicine Reviews, vol. 18, no. 3, pp. 273-282, 2014.

[27] C. Alexandre, A. Latremoliere, A. Ferreira et al., "Decreased alertness due to sleep loss increases pain sensitivity in mice," Nature Medicine, vol. 23, no. 6, pp. 768-774, 2017.

[28] P. H. Finan, B. R. Goodin, and M. T. Smith, "The association of sleep and pain: an update and a path forward," The Journal of Pain, vol. 14, no. 12, pp. 1539-1552, 2013.

[29] S. M. Bigatti, A. M. Hernandez, T. A. Cronan, and K. L. Rand, "Sleep disturbances in fibromyalgia syndrome: relationship to pain and depression," Arthritis \& Rheumatism, vol. 59, no. 7, pp. 961-967, 2008.

[30] M. H. Bromberg, K. M. Gil, and L. E. Schanberg, "Daily sleep quality and mood as predictors of pain in children with juvenile polyarticular arthritis," Health Psychology, vol. 31, no. 2, pp. 202-209, 2012.

[31] A. S. Lewandowski, T. M. Palermo, S. De la Motte, and R. Fu, "Temporal daily associations between pain and sleep in 
adolescents with chronic pain versus healthy adolescents," Pain, vol. 151, no. 1, pp. 220-225, 2010.

[32] N. K. Tang, C. E. Goodchild, A. N. Sanborn, J. Howard, and P. M. Salkovskis, "Deciphering the temporal link between pain and sleep in a heterogeneous chronic pain patient sample: a multilevel daily process study," Sleep, vol. 35 , no. 5, pp. 675-687a, 2012.

[33] B. S. Chhangani, T. A. Roehrs, E. J. Harris et al., "Pain sensitivity in sleepy pain-free normals," Sleep, vol. 32 , no. 8 , pp. 1011-1017, 2009.

[34] N. Mehta, I. Bucior, S. Bujanover, R. Shah, and A. Gulati, "Relationship between pain relief, reduction in pain-associated sleep interference, and overall impression of improvement in patients with postherpetic neuralgia treated with extended-release gabapentin," Health and Quality of Life Outcomes, vol. 14, p. 54, 2016.

[35] A. Vinik, B. Emir, R. Cheung, and E. Whalen, "Relationship between pain relief and improvements in patient function/ quality of life in patients with painful diabetic peripheral neuropathy or postherpetic neuralgia treated with pregabalin," Clinical Therapeutics, vol. 35, no. 5, pp. 612-623, 2013.

[36] J. I. Gerhart, J. W. Burns, K. M. Post et al., "Relationships between sleep quality and pain-related factors for people with chronic low back pain: tests of reciprocal and time of day effects," Annals of Behavioral Medicine, vol. 51, no. 3, pp. 365-375, 2017.

[37] D. J. Buysse, C. F. Reynolds 3rd, T. H. Monk, S. R. Berman, and D. J. Kupfer, "The Pittsburgh Sleep Quality Index: a new instrument for psychiatric practice and research," Psychiatry Research, vol. 28, no. 2, pp. 193-213, 1989.

[38] X. C. Liu, M. Q. Tang, L. Hu et al., "Reliability and validity of Pittsburgh sleep quality index," Chinese Journal of Psychiatry, vol. 29, no. 2, pp. 103-107, 1996.

[39] A. C. Parrott and I. Hindmarch, "Factor analysis of a sleep evaluation questionnaire," Psychological Medicine, vol. 8, no. 2, pp. 325-329, 1978.

[40] N. Zisapel and M. Laudon, "Subjective assessment of the effects of CNS-active drugs on sleep by the Leeds sleep evaluation questionnaire: a review," Human Psychopharmacology, vol. 18, no. 1, pp. 1-20, 2003.

[41] H. Choi, S. Kim, B. Kim, and I. Kim, "Psychometric properties of the Korean versions of three sleep evaluation questionnaires," Clinical Nursing Research, vol. 24, no. 5, pp. 526-538, 2015.

[42] R. Tarrasch, M. Laudon, and N. Zisapel, "Cross-cultural validation of the Leeds sleep evaluation questionnaire (LSEQ) in insomnia patients," Human Psychopharmacology, vol. 18, no. 8, pp. 603-610, 2003.

[43] D. J. Buysse, "Sleep health: can we define it? Does it matter?" Sleep, vol. 37, no. 1, pp. 9-17, 2014.

[44] X. Chen, B. Gelaye, and M. A. Williams, "Sleep characteristics and health-related quality of life among a national sample of American young adults: assessment of possible health disparities," Quality of Life Research, vol. 23, no. 2, pp. 613-625, 2014.

[45] X. Liu, J. Chen, J. Zhou et al., "The relationship between the number of daily health-related behavioral risk factors and sleep health of the elderly in China," International Journal of Environmental Research and Public Health, vol. 16, no. 24, 2019.

[46] L. Lu, S. B. Wang, W. Rao et al., "The prevalence of sleep disturbances and sleep quality in older Chinese adults: a comprehensive meta-analysis," Behavioral Sleep Medicine, vol. 17, no. 6, pp. 683-697, 2019.

[47] J. A. Rabbitts, C. Zhou, A. Narayanan, and T. M. Palermo, "Longitudinal and temporal associations between daily pain and sleep patterns after major pediatric surgery," The Journal of Pain, vol. 18, no. 6, pp. 656-663, 2017.

[48] E. Koffel, S. M. McCurry, M. T. Smith, and M. V. Vitiello, "Improving pain and sleep in middle-aged and older adults: the promise of behavioral sleep interventions," Pain, vol. 160, no. 3, pp. 529-534, 2019.

[49] M. N. Bomalaski, E. S. Claflin, W. Townsend, and M. D. Peterson, "Zolpidem for the treatment of neurologic disorders: a systematic review," JAMA Neurology, vol. 74, no. 9, pp. 1130-1139, 2017.

[50] F. Kong, G. Liu, and J. Xu, "Pharmacological agents for improving sleep quality at high altitude: a systematic review and meta-analysis of randomized controlled trials," Sleep Medicine, vol. 51, pp. 105-114, 2018.

[51] L. P. Malver, A. Brokjaer, C. Staahl, C. Graversen, T. Andresen, and A. M. Drewes, "Electroencephalography and analgesics," British Journal of Clinical Pharmacology, vol. 77, no. 1, pp. 72-95, 2014.

[52] L. Wu, S. Wang, Y. Wang, K. Zhang, J. Bai, and J. Zheng, "Prediction of hemodynamic reactivity by electroencephalographically derived pain threshold index in children undergoing general anesthesia: a prospective observational study," Journal of Pain Research, vol. 12, pp. 3245-3255, 2019.

[53] Y. B. Wu, "Extraction of objective and quantitative indexes of pain, anxiety, depression and other brain function states from electroencephalogram," China Medical Engineering, vol. 25, no. 4, pp. 1-7, 2017.

[54] M. F. Bjurström and M. R. Irwin, "Perioperative pharmacological sleep-promotion and pain control: a systematic review," Pain Practice, vol. 19, no. 5, pp. 552-569, 2019.

[55] H. Shakya, D. Wang, K. Zhou, Z. Y. Luo, S. Dahal, and Z. K. Zhou, "Prospective randomized controlled study on improving sleep quality and impact of zolpidem after total hip arthroplasty," Journal of Orthopaedic Surgery and Research, vol. 14, no. 1, p. 289, 2019.

[56] L. Gong, Z. Wang, and D. Fan, "Sleep quality effects recovery after total knee arthroplasty (TKA) - a randomized, doubleblind, controlled study," The Journal of Arthroplasty, vol. 30, no. 11, pp. 1897-1901, 2015.

[57] G. W. Sun, Y. L. Yang, X. B. Yang et al., "Preoperative insomnia and its association with psychological factors, pain and anxiety in Chinese colorectal cancer patients," Supportive Care in Cancer, vol. 28, no. 6, pp. 2911-2919, 2020.

[58] W. Wang, Z. Dong, X. Zhang, W. Li, P. Li, and X. Chen, "Dietary and the risk of sporadic colorectal cancer in China: a case-control study," Iranian Journal of Public Health, vol. 47, no. 9, pp. 1327-1335, 2018.

[59] S. Sikandar and A. H. Dickenson, "Visceral pain: the ins and outs, the ups and downs," Current Opinion in Supportive and Palliative Care, vol. 6, no. 1, pp. 17-26, 2012.

[60] A. M. Drewes, A. E. Olesen, A. D. Farmer, E. Szigethy, V. Rebours, and S. S. Olesen, "Gastrointestinal pain," Nature Reviews Disease Primers, vol. 6, no. 1, p. 1, 2020.

[61] S. Chokhavatia, E. S. John, M. B. Bridgeman, and D. Dixit, "Constipation in elderly patients with noncancer pain: focus on opioid-induced constipation," Drugs \& Aging, vol. 33, no. 8 , pp. $557-574,2016$.

[62] A. Herrero Babiloni, B. P. De Koninck, G. Beetz, L. De Beaumont, M. O. Martel, and G. J. Lavigne, "Sleep and pain: recent insights, mechanisms, and future directions in the investigation of this relationship," Journal of Neural Transmission, vol. 127, no. 4, pp. 647-660, 2020.

[63] M. T. Smith, R. R. Edwards, U. D. McCann, and J. A. Haythornthwaite, "The effects of sleep deprivation on 
pain inhibition and spontaneous pain in women," Sleep, vol. 30, no. 4, pp. 494-505, 2007.

[64] S. Iacovides, K. George, P. Kamerman, and F. C. Baker, "Sleep fragmentation hypersensitizes healthy young women to deep and superficial experimental pain," The Journal of Pain, vol. 18, no. 7, pp. 844-854, 2017.

[65] N. Eichhorn, R. D. Treede, and S. Schuh-Hofer, "The role of sex in sleep deprivation related changes of nociception and conditioned pain modulation," Neuroscience, vol. 387, pp. 191-200, 2018.

[66] M. T. Smith Jr, B. Remeniuk, P. H. Finan et al., "Sex differences in measures of central sensitization and pain sensitivity to experimental sleep disruption: implications for sex differences in chronic pain," Sleep, vol. 42, no. 2, Article ID zsy209, 2019.

[67] A. I. Basbaum, D. M. Bautista, G. Scherrer, and D. Julius, "Cellular and molecular mechanisms of pain," Cell, vol. 139, no. 2, pp. 267-284, 2009.

[68] J. M. Zhang, "An J. Cytokines, inflammation, and pain," International Anesthesiology Clinics, vol. 45, no. 2, pp. 27-37, 2007.

[69] J. M. Mullington, N. S. Simpson, H. K. Meier-Ewert, and M. Haack, "Sleep loss and inflammation," Best Practice \& Research Clinical Endocrinology \& Metabolism, vol. 24, no. 5, pp. 775-784, 2010.

[70] M. R. Irwin, R. Olmstead, and J. E. Carroll, "Sleep disturbance, sleep duration, and inflammation: a systematic review and meta-analysis of cohort studies and experimental sleep deprivation," Biological Psychiatry, vol. 80, no. 1, pp. 40-52, 2016.

[71] J. C. Ong, N. B. Cardé, J. J. Gross, and R. Manber, “A twodimensional approach to assessing affective states in good and poor sleepers," Journal of Sleep Research, vol. 20, no. 4, pp. 606-610, 2011.

[72] S. A. Janssen, "Negative affect and sensitization to pain," Scandinavian Journal of Psychology, vol. 43, no. 2, pp. 131-137, 2002.

[73] V. Roman, R. Hagewoud, P. G. Luiten, and P. Meerlo, "Differential effects of chronic partial sleep deprivation and stress on serotonin-1A and muscarinic acetylcholine receptor sensitivity," Journal of Sleep Research, vol. 15, no. 4, pp. 386-394, 2006.

[74] P. K. Eide, "Wind-up and the NMDA receptor complex from a clinical perspective," European Journal of Pain, vol. 4, no. 1, pp. 5-15, 2000.

[75] R. Staud, C. J. Vierck, M. E. Robinson, and D. D. Price, "Effects of the N-methyl-D-aspartate receptor antagonist dextromethorphan on temporal summation of pain are similar in fibromyalgia patients and normal control subjects," The Journal of Pain, vol. 6, no. 5, pp. 323-332, 2005.

[76] C. Kopp, F. Longordo, J. R. Nicholson, and A. Lüthi, "Insufficient sleep reversibly alters bidirectional synaptic plasticity and NMDA receptor function," Journal of Neuroscience, vol. 26, no. 48, pp. 12456-12465, 2006.

[77] S. C. Holst, A. Sousek, K. Hefti et al., "Cerebral mGluR5 availability contributes to elevated sleep need and behavioral adjustment after sleep deprivation," Elife, vol. 6, 2017.

[78] K. Hefti, S. C. Holst, J. Sovago et al., "Increased metabotropic glutamate receptor subtype 5 availability in human brain after one night without sleep," Biological Psychiatry, vol. 73, no. 2, pp. 161-168, 2013.

[79] N. D. Volkow, D. Tomasi, G. J. Wang et al., "Evidence that sleep deprivation downregulates dopamine D2R in ventral striatum in the human brain," Journal of Neuroscience, vol. 32, no. 19 , pp. 6711-6717, 2012.

[80] M. Moradi, M. Yazdanian, and A. Haghparast, "Role of dopamine D2-like receptors within the ventral tegmental area and nucleus accumbens in antinociception induced by lateral hypothalamus stimulation," Behavioural Brain Research, vol. 292, pp. 508-514, 2015.

[81] C. G. Pick, Y. Chernes, T. Rigai, K. C. Rice, and S. Schreiber, "The antinociceptive effect of zolpidem and zopiclone in mice," Pharmacology, Biochemistry, and Behavior, vol. 81, no. 3, pp. 417-423, 2005.

[82] E. H. Yu, D. H. Tran, S. W. Lam, and M. G. Irwin, "Remifentanil tolerance and hyperalgesia: short-term gain, longterm pain?" Anaesthesia, vol. 71, no. 11, pp. 1347-1362, 2016. 